\title{
WARPED PERSPECTIVES IN TIME-FREQUENCY ANALYSIS
}

\author{
Richard G. Baraniuk* \\ Department of Electrical and Computer Engineering \\ Rice University \\ Houston, TX 77251-1892, USA
}

\begin{abstract}
Unitary similarity transformations furnish a simple yet powerful vehicle for generating new classes of joint distributions based on concepts different from time, frequency, and scale. These new signal representations focus on the critical characteristics of large classes of signals and, hence, prove useful for representing and processing signals that are not well matched by current techniques. In particular, we construct distributions to match almost any one-to-one group delay or instantaneous frequency characteristic. Amazingly, all distributions that have been used to illustrate more complicated generalized distribution design techniques can be generated using the unitary transformation method.
\end{abstract}

\section{INTRODUCTION}

Recent advances in nonstationary signal analysis can be attributed primarily to the development of two sets of analysis tools: Cohen's class of joint time-frequency distributions (TFDs), which contains the short-time Fourier transform and the Wigner distribution [1], and the affine class of joint timescale distributions (TSDs), which contains the continuous wavelet transform [2]. While these representations are natural for signals containing pulse, sinusoidal, linear chirping, or self-similar components, there exist many other signal classes that are not well described in terms of the time, frequency, or scale variables. For instance, many applications (radar, sonar, geophysics, optical communications, among others) involve dispersed pulses $\delta(m(x)-t)$ or chirping tones $e^{j 2 \pi f m(x)}$ to which neither TFDs nor TSDs are matched. Matching these types of signals requires new joint distributions with different instantaneous frequency and group delay localization properties. Historically, new distributions have been developed in a piecemeal fashion, with new ones being created for each new signal class of interest $[3,4,5]$.

In this paper, we introduce an alternative methodology that generalizes TFDs and TSDs to infinite classes of new joint representations, each of which is perfectly matched to a specific class of signals $[6,7]$. The key to our approach lies in a special family of "basis changing" operators - the unitary transformations - which convert traditional systems into new systems with different properties. Figure 1 illustrates the general scheme: an arbitrary conventional signal processing tool $\mathcal{P}$ (TFD or TSD in this paper) is cradled between two unitary transformations $\mathcal{U}$ and $\mathcal{V}$ to form the unitarily equivalent tool $\mathcal{V P U}$.

The transformations $U$ and $\mathcal{V}$ change the fundamental coordinate system of $\mathcal{P}$, mapping familiar concepts such as time,

*This work was supported by the NSF, grant no. MIP-9457438, and by the State of Texas, grant no. TX-ATP 003604-002. frequency, and scale to new concepts more natural for analyzing certain types of signals. The effect of the preprocessing $\mathcal{U}$ on TFDs and TSDs has been documented in $[6,7]$; we review these results in the next section. The remaining sections concentrate on the surprisingly powerful role that the postprocessing $\mathcal{V}$ can play in generalizing TFDs and TSDs, from TFDs and TSDs matched to specific instantaneous frequency or group delay laws (Section 3 ) to distributions of almost arbitrary variables (Section 4 ).

\section{WARPED DISTRIBUTIONS THROUGH UNITARY EQUIVALENCE}

The unitary equivalence concept was introduced to signal processing in $[6,7]$. Operator methods simplify its treatment considerably. To a physical quantity denoted by the variable $a$, we can associate two operator representations: one Hermetian, $A$, and one unitary, $\mathcal{A}_{\alpha}[1,7,8]$. The eigenfunctions $\mathrm{e}_{a}^{A}$ of $A$ form the basis for the $A$-Fourier transform $\mathrm{F}_{A}$, with $\left(\mathbf{F}_{A} s\right)(a) \stackrel{\text { def }}{=} \int s(x) \mathrm{e}_{a}^{A *}(x) d x$. Due to its covariance to the unitary representation $\mathcal{A}_{\alpha}\left(\left(\mathbf{F}_{A} \mathcal{A}_{\alpha} s\right)(a)=\left(\mathbf{F}_{A} s\right)(a \bullet \alpha)\right.$, where $\bullet$ denotes the group operation induced by $\mathcal{A}_{\alpha_{1}} \mathcal{A}_{\alpha_{2}}=$ $\left.\mathcal{A}_{\alpha_{1} \bullet \alpha_{2}}[9,10]\right)$, we say that $\mathbf{F}_{A}$ measures the "a" content of the time signal $s$.

Time and frequency are fundamental concepts for both TFDs and TSDs. To the time variable $t$, we associate the Hermetian and unitary operator representations $(T s)(x)=$ $x s(x)$ and $\left(\mathcal{T}_{t} s\right)(x)=s(x-t)$, respectively. The time eigenfunctions $\mathbf{e}_{t}^{T}(x)=\delta(x-t)$ induce the time transform $\left(\mathbf{F}_{T} s\right)(t)=s(t)$. To the frequency variable $f$, we associate the Hermetian and unitary operator representations $(F s)(x)=\frac{1}{2 \pi j} \dot{s}(x)$ and $\left(\mathcal{F}_{f} s\right)(x)=e^{j 2 \pi f x} s(x)$, respectively. The frequency eigenfunctions $\mathrm{e}_{f}^{F}(x)=e^{j 2 \pi f x}$ induce the frequency (Fourier) transform $\left(\mathbf{F}_{F} s\right)(f)=S(f)$. We will usually omit the subscript $F$ from the Fourier transform.

Two operators $\mathcal{A}$ and $\tilde{\mathcal{A}}$ are said to be unitarily equivalent if we can write $\widetilde{\mathcal{A}}=\mathcal{U}^{-1} \mathcal{A} \mathcal{U}$, with $\mathcal{U}$ a unitary transformation. Preprocessing a system $\mathcal{P}$ with a unitary transform $\mathcal{U}$ (as in Fig. 1, omitting $\mathcal{V}$ ) maps the variables associated with the Hermetian or unitary operators $\mathcal{A}, \mathcal{B}, \cdots$ to the variables associated with the unitarily equivalent operators $\widetilde{\mathcal{A}}=\mathcal{U}^{-1} \mathcal{A} \mathcal{U}, \widetilde{\mathcal{B}}=\mathcal{U}^{-1} \mathcal{B} U, \cdots[6,7]$. Remapped signal transforms measure these new quantities; the transform $F_{\widetilde{A}}=F_{A} U$ is obtained by projecting the signal onto the unitarily equivalent eigenfunctions $\mathbf{e}_{a}^{\tilde{A}}=U^{-1} \mathrm{e}_{a}^{A}$. Each choice of $\mathcal{U}$ generates a system $\mathcal{P} U$ matched to a radically different set of physical quantities. 
The time domain axis warping operators,

$$
(U s)(x)=|\dot{w}(x)|^{1 / 2} s[w(x)]
$$

with $w$ a smooth one-to-one function, form a large and indispensable subclass of unitary transformations. Composition with the Fourier transform yields the frequency domain axis warping $\mathbf{F}^{-1} \mathcal{U} \mathbf{F}$. The great utility of warping operators prompts the following abuse of terminology: even if $\mathcal{U}$ is not of the form (1), we will refer to the unitarily equivalent preprocessed system $\mathcal{P U}$ as a "warped system" and refer to the operators $\widetilde{\mathcal{A}}, \widetilde{\mathcal{B}}, \cdots$ as "warped versions" of $\mathcal{A}, \mathcal{B}, \cdots$

In this paper, we will concentrate on the covariance, marginal, and localization properties of warped TFDs and TSDs; for a detailed discussion of other properties, see [6, 7]. Denoting a TFD from Cohen's class using the operator notation $(\mathcal{C} s)(\tau, \nu)$, we define a $\mathcal{U}$-Cohen's class as the set of prewarped distributions $(\mathcal{C U} s)(\tau, \nu)$. Covariance to the time and frequency shift operators $\mathcal{T}$ and $\mathcal{F}$ and marginals of time $\mathbf{F}_{T}$ and frequency $\mathbf{F}_{F}$ make the TFDs of Cohen's class ideal for studying signals whose components resemble the time and frequency eigenfunctions (pulses and sinusoids) [1]. Likewise, simple substitution demonstrates the covariance to $\widetilde{\mathcal{T}}$ and $\widetilde{\mathcal{F}}$

$$
\left(\mathcal{C U} \tilde{\mathcal{F}}_{m} \tilde{T}_{n} s\right)(\tau, \nu)=(\mathcal{C U} s)(\tau-n, \nu-m)
$$

and marginals of warped time and warped frequency

$$
\begin{aligned}
& \int(\mathcal{C U} s)(\tau, \nu) d \nu=\left|\left(\mathcal{U}_{s}\right)(\tau)\right|^{2}=\left|\left(\mathbf{F}_{\widetilde{T}} s\right)(\tau)\right|^{2} \\
& \int(\mathcal{C U} s)(\tau, \nu) d \tau=|(\mathbf{F} \mathcal{U} s)(\nu)|^{2}=\left|\left(\mathbf{F}_{\widetilde{F}} s\right)(\nu)\right|^{2}
\end{aligned}
$$

that make $\mathcal{U}$-Cohen's class distributions ideal for studying signals whose components resemble the warped time and frequency eigenfunctions.

The freedom of choice of prewarping transformation $\mathcal{U}$ unleashes an endless variety of $\mathcal{U}$-Cohen's classes, each of which perfectly matches a particular signal class. For example, time domain axis warping transformations of the form (1) convert Cohen's class TFDs to distributions matched simultaneously to the dispersed pulse $\delta(m(x)-t)|\dot{m}(x)|^{1 / 2}$ and the chirping tone $e^{j 2 \pi f m(x)}|\dot{m}(x)|^{1 / 2}$, with $m=w^{-1}$. These elementary signal components are concentrated along a constant group delay and along the chirping instantaneous frequency $\dot{m}(x)$, respectively, and are therefore best represented in terms of neither time-frequency nor time-scale coordinates.

The prehyperbolic class of [3] provides a concrete example of a frequency domain axis warping. The transformation $\mathcal{U}_{\text {log }} \stackrel{\text { def }}{=} \mathrm{F}^{-1} \mathcal{U}_{1} \mathbf{F}$, with $\left(\mathcal{U}_{1} s\right)(x) \stackrel{\text { def }}{=} e^{x / 2} s\left(e^{x}\right)$, maps the frequency and time coordinates of Cohen's class to $\mathcal{U}_{\text {log }}$-Cohen's class coordinates of scale and "hyperbolic time shift" [3], a dispersive time shift that translates high frequency signal components less than low frequency signal components. The covariance and marginal properties of $\mathcal{U}_{\log }-$ Cohen's class distributions match signals resembling the analytic eigenfunctions

$$
\begin{aligned}
& \left(\mathcal{U}_{\log }^{-1} \mathbf{e}_{h}^{T}\right)(x)=F_{\omega \mapsto x}^{-1} \frac{1}{\sqrt{\omega}} e^{j 2 \pi h \log \omega} \\
& \left(\mathcal{U}_{\log }^{-1} \mathbf{e}_{d}^{F}\right)(x)=e^{d / 2} e^{j 2 \pi e^{d} x} .
\end{aligned}
$$

The terms hyperbolic time shift and hyperbolic class stem from the localization of the $\mathcal{U}_{\log }^{-1} \mathrm{e}_{h}^{T}$ along hyperbolas in the time-frequency plane. Figures 2 and 3 illustrate the Wigner TFD and the $\mathcal{U}_{\log }-W$ igner (Altes-Marinovich " $Q$ " [3]) distribution of the sum of two such hyperbolic chirps. The dramatic increase in clarity from Fig. 2 to Fig. 3 is indicative of the improvement obtainable by matching the analysis coordinate system to the data. For more $\mathcal{U}$-Cohen's class examples, including distributions of "power time" and "chirp rate," see $[6,7]$.

The TSDs of the affine class offer an alternative analysis to that of Cohen's class [2]. TSDs are covariant to time shifts $\mathcal{T}$ and scale changes $\mathcal{D}$ and are based on marginals of time $\mathbf{F}_{T}$ and inverse frequency $\left(\mathbf{F}_{f_{0} / F} s\right)(r)=(\mathbf{F} s)\left(f_{0} / r\right)$ [8]. Just as above, we can define a $U$-affine class $[6,7]$ containing distributions of the form $(\mathcal{G U} s)(\tau, r)$, where $\left(\mathcal{G}_{s}\right)(\tau, r)$ denotes a generic TSD. As a simple example, consider the frequencydomain power warping introduced in [5]: $\mathcal{U}_{c} \stackrel{\text { def }}{=} \mathbf{F}^{-1} \mathcal{U}_{2} \mathbf{F}$ with $\mathcal{U}_{2}$ of the form $(1)$ and $w(x)=|x|^{1 / c} \operatorname{sgn}(x)$. The $U_{c}-$ affine class coincides with the power class [4] of "chirp time" and scale distributions and contains the scale-shear metaplectic transform from [11]. The commutation of $\mathcal{U}_{c}$ with the scale operator $[4,5]$ lends this warped class to studying scale-invariant, power-law signals.

\section{DOUBLY WARPED DISTRIBUTIONS}

Up to this point, we have concentrated exclusively on unitary preprocessing transformations; however, the postprocessing transformation $\mathcal{V}$ from Fig. 1 also merits careful consideration. Postprocessing adds considerable richness to the already powerful $\mathcal{U}$-Cohen's and $\mathcal{U}$-affine classes. We define a $\mathcal{V U}$-Cohen's class as the set of distributions $\mathcal{V} U_{s}$, with $\mathcal{C} s$ a Cohen's class TFD, $\mathcal{U}$ a unitary preprocessing signal transformation, and $\mathcal{V}: L^{2}\left(\mathbb{R}^{2}\right) \rightarrow L^{2}\left(\mathbb{R}^{2}\right)$ a unitary postprocessing transformation [7]. Note that $\mathcal{V}$ operates on the warped time-frequency plane. A $\mathcal{V} U$-affine class is defined similarly.

3.1 Warping back to time-frequency. While warped TFDs show considerable promise for matching signals noncoincident with constant or linear group delays and instantaneous frequencies, certain applications demand representations with time and frequency axes rather than warped time and warped frequency axes. Fortunately, we can use $\mathcal{V}$ to invert the action of $\mathcal{U}$ on the warped time-frequency plane, warping the $(\tau, \nu)$ axes of $\mathcal{U}$-Cohen's class distributions to new axes $(p, q)$ providing correct time-frequency localization. While these $\mathcal{V} \mathcal{U}$-Cohen's class distributions measure timefrequency content in signals, they remain matched to the same group delay or instantaneous frequency characteristics as the corresponding $\mathcal{U}$-Cohen's class.

Our procedure for inverting the effect of $U$ is best interpreted graphically on the warped time-frequency plane [7] A $\mathcal{U}$-Cohen's class distribution $c \mathcal{c} s$ maps the time eigenfunction $\mathbf{e}_{p}^{T}(x)=\delta(x-p)$ localized at time $p$ and the fre quency eigenfunction $\mathrm{e}_{q}^{F}(x)=e^{j 2 \pi q x}$ localized at frequency $q$ to distributions localized on curves in the $(\tau, \nu)$ plane. The equations of these curves can be obtained from the $\mathcal{U}$-Cohen's class analogues of the time-frequency concepts of group delay and instantaneous frequency. Modifying the usual definitions of these quantities [1] to account for the preprocessing by $\mathcal{U}$, we obtain a curve for the localization of $\mathbf{e}_{p}^{T}$ (its " $\mathcal{U}$-group delay")

$$
\rho(\nu, p) \stackrel{\text { def }}{=}-\frac{1}{2 \pi} \frac{\partial}{\partial \nu} \arg \left(\mathbf{F} \mathcal{U} \mathbf{e}_{p}^{T}\right)(\nu)
$$

and a curve for the localization of $\mathbf{e}_{q}^{F}$ (its " $\mathcal{U}$-instantaneous frequency")

$$
\theta(\tau, q) \stackrel{\text { def }}{=} \frac{1}{2 \pi} \frac{\partial}{\partial \tau} \arg \left(\mathcal{U} \mathrm{e}_{q}^{F}\right)(\tau)
$$


If $\mathcal{V}$ reparameterizes the axes of the $(\tau, \nu)$ plane in terms of these curves, then $\mathbf{e}_{p}^{T}$ and $\mathbf{e}_{q}^{F}$ will be localized along straight lines at time $p$ and frequency $q$, and correct time-frequency localization will result, albeit with nonuniform resolution. The requisite reparameterization is given by

$$
(\mathcal{V C U} s)(p, q)=(\mathcal{C U} s)(\underline{\tau}(p, q), \underline{\nu}(p, q)),
$$

where the functions $\tau(p, q)$ and $\underline{\nu}(p, q)$ denote the solution (when one exists) to the system of equations $\tau=\rho(\nu, p)$, $\nu=\theta(\tau, q)$ for $\tau$ and $\nu$ in terms of $p$ and $q$. The reverse reparameterization is also useful and is given by

$$
(\mathcal{V C U} s)(\underline{p}(\tau, \nu), \underline{q}(\tau, \nu))=(\mathcal{C U} s)(\tau, \nu),
$$

where the functions $\underline{p}(\tau, \nu)$ and $\underline{q}(\tau, \nu)$ denote the solution to $\tau=\rho(\nu, p), \nu=\theta(\tau, q)$ in terms of $\tau$ and $\nu .^{1}$

In effect, $\mathcal{V}$ changes the geometry of a $\mathcal{U}$-Cohen's class so that: (1) impulses and sinusoids lie concentrated along lines parallel to the $(p, q)$ axes, and (2) the warped eigenfunctions lie concentrated along their true instantaneous frequencies or group delays. Reconsidering the $\mathcal{U}_{1 \mathrm{og}}$-Cohen's class example from the previous section, the formulas in Footnote 1 below give $\underline{\tau}(p, q)=p q, \underline{\nu}(p, q)=\log q$ as the re quired axis reparameterization. Making these substitutions, as $\left(\mathcal{V}_{\log } \mathcal{C} \mathcal{U}_{\log } s\right)(p, q)=\left(\mathcal{C} \mathcal{U}_{\log } s\right)(p q, \log q)$, yields the hyperbolic class of TFDs [3]. The hyperbolic geometry of the warping $V_{\log }$ is evident in the $\mathcal{V}_{\log } \mathcal{U}_{\log }$-Wigner distribution (Q TFD of Altes [3]) of the sum of two hyperbolic chirp functions given in Fig. 4.

Comparison of Figs. 2 and 4 reveals the salient feature distinguishing Cohen's class from a $\mathcal{V U}$-Cohen's class, both of which measure the time-frequency content of signals: the geometry of Cohen's class perfectly localizes the impulses $\mathbf{e}_{t}^{T}$ and sinusoids $\mathbf{e}_{f}^{F}$, while the geometry of a $\mathcal{V U}$-Cohen's class perfectly localizes the functions $\mathcal{U}^{-1} \mathbf{e}_{t}^{T}$ and $U^{-1} \mathbf{e}_{f}^{F}$. Thus, for example, the hyperbolic class contains the TFDs of choice for studying in time-frequency signals localized along either constant instantaneous frequencies or hyperbolic group delays.

While $\mathcal{V U}$-Cohen's class distributions remain covariant to the warped time and frequency operators $\widetilde{\mathcal{T}}$ and $\widetilde{\mathcal{F}}$, translation covariance is replaced by a different sort; see [7] for the details. The distortion introduced by $\mathcal{V}$ also changes the marginal properties from the straight line integrals of (2) to the path integrals

$$
\begin{aligned}
& \int(\mathcal{V} \mathcal{C U} s)(\underline{p}(p, q), \underline{q}(p, q)) d q=|(\mathcal{U} s)(p)|^{2} \\
& \int(\mathcal{V} \mathcal{C U} s)(\underline{p}(p, q), \underline{q}(p, q)) d p=\left|\left(\mathrm{F} U_{s}\right)(q)\right|^{2} .
\end{aligned}
$$

In the hyperbolic class, for example, we integrate along hyperbolas in (6) and parallel to the $p$ axis in (7).

3.2 Warp synthesis. Up to now we have considered how particular choices of $\mathcal{U}$ and $\mathcal{V}$ transform Cohen's class TFDs into distributions matched to particular group delay or instantaneous frequency characteristics. We now consider the inverse problem of warp synthesis [7], which can be stated as:

\footnotetext{
${ }^{1}$ For preprocessing transformations $U$ of the warp type (1), these functions reduce to $\underline{\tau}(p, q)=w^{-1}(p), \underline{\nu}(p, q)=q \dot{w}\left(w^{-1}(p)\right)$, $\underline{p}(\tau, \nu)=w(\tau), \underline{q}(\tau, \nu)=\frac{\nu}{\dot{w}(\tau)}$, while for frequency axis warping transformations of the form $F^{-1} \mathcal{U} \mathbf{F}$, the functions reduce to $\underline{\tau}(p, q)=p \dot{w}\left(w^{-1}(q)\right), \underline{\nu}(p, q)=w^{-1}(q), \underline{p}(\tau, \nu)=\frac{\tau}{\dot{w}(\nu)}$, $\underline{q}(\tau, \nu)=w(\nu)$. The unitarity of $\mathcal{V}$ on $L^{2}\left(\mathbf{R}^{2}\right)$ is readily verified.
}

find the unitary operator $\mathcal{U}$ giving a $\mathcal{V} U$-Cohen's class that perfectly localizes two types of signals - those whose group delay lies along the curve $\gamma_{2}(q)$ in the $(p, q)$ plane and those whose instantaneous frequency lies along the curve $\gamma_{1}(p)$ in the $(p, q)$ plane.

When a solution exists to the warp synthesis problem, it can be found by reversing the procedure of Section 3.1. The two paths $\gamma_{1}$ and $\gamma_{2}$ specify $\rho(\nu, p)$ and $\theta(\tau, q)$; integration yields functions from which the form of $\mathcal{U}$ can be inferred. Clearly, a key consideration is the invertibility of the system of equations (4), (5). The solution is straightforward when $\mathcal{U}$ is constrained to be a time or frequency axis warping, however. In the time domain case, with $\mathcal{U}$ of the form (1), only the instantaneous frequency localization can be adjusted, the group delay localization being fixed along lines parallel to the frequency axis. Given a desired, one-to-one instantaneous frequency function $\gamma_{1}(p)$, the corresponding warping function $w$ for $\mathcal{U}$ is given by the inverse of the indefinite integral of $\gamma_{1}$; that is, $w=z^{-1}, z(u)=\int \gamma_{1}(u) d u$. In the frequency domain case, for operators of the form $\mathbf{F}^{-1} \mathcal{U} \mathbf{F}$ with $\mathcal{U}$ from (1), the calculation is identical and yields TFDs that localize along group delays of the form $\gamma_{2}(g)$.

This design procedure, while quite simple, works for signal sets concentrated along arbitrary one-to-one group delays or instantaneous frequencies. For example, a desire to localize signals having hyperbolic group delays would prompt the selection of $\gamma_{2}(q)=\frac{c}{q}$. Integration of $\gamma_{2}$ followed by inversion yields the warping function $w(x)=e^{x / c}$ and the preprocessing operator $\mathbf{F}^{-1} \mathcal{U}_{1} \mathbf{F}=\mathcal{U}_{\log }$. The prehyperbolic and hyperbolic classes of hyperbolic time and scale distributions result from this processing.

The TSDs of the affine class can also be doubly warped to generate classes of distributions based on the warped operators $\widetilde{T}$ and $\widetilde{\mathcal{D}}$ yet indicating true time-scale (or, more precisely, time and inverse frequency) content. Space considerations limit a complete discussion; see [7] for more details.

\section{DOUBLY WARPED DISTRIBUTIONS FOR "ALMOST ARBITRARY" VARIABLES}

Unitary preprocessing can map the operator pair $T, F$ fundamental to Cohen's class TFDs to a wide variety of unitarily equivalent operator pairs $\widetilde{T}, \widetilde{F}$ generating $\mathcal{U}$-Cohen's classes. However, the "structure preserving" nature of unitary transformations constrains the relationship between $\widetilde{T}$ and $\widetilde{F}$ to be the same as that between $T$ and $F{ }^{2}$ Thus, once we select a $U$ to localize on a particular group delay, we also fix the instantaneous frequency localization of the $\mathcal{U}$-Cohen's class. Equivalently, specification of $\mathcal{U}$ locks in both marginal properties simultaneously. For example (see (2), (3), and (6), (7)), while the $\mathcal{U}_{\mathrm{IOg}}$-Cohen's class possesses a potentially desirable Mellin transform for one marginal, we are forced to accept the potentially undesirable $\log$ frequency transform for the other. Distributions with Mellin and time or frequency marginals cannot be generated as a $U$-Cohen's class.

To construct distributions based on operator pairs $A, B$ with $[A, B] \neq[T, F]$, we have been compelled in the past to resort to more complicated general methods [8, 9]. However, with a clever choice of prewarping and postwarping transformations we can generate joint distributions for a very

\footnotetext{
${ }^{2}$ Using the commutator $[A, B]=A B-B A$ as a measure of structural similarity, we have that $[\widetilde{T}, \widetilde{F}]=\mathcal{U}^{-1}[T, F] \mathcal{U}=[T, F]$, where $[T, F]=\frac{1}{j 2 \pi} \mathcal{I}$ with $\mathcal{I}$ the identity operator.
} 
large class of variables (hence our usage of "almost arbitrary" above). This new double warping procedure differs fundamentally from that of the previous section.

Theorem: Let $A, B$ be a Hermetian operator pair such that $A=\mathcal{Z}^{-1} T \mathcal{Z}$ and $B=\mathcal{U}^{-1} F \mathcal{U}$, where $\mathcal{U}$ and $\mathcal{Z}$ are unitary transformations having a composition $\mathcal{Z} \mathcal{U}^{-1}$ that is a smooth axis warping operator of the form $s(x) \mapsto|\dot{v}(x)|^{1 / 2} s[v(x)]$. Then joint distributions having $\mathbf{F}_{A}, \mathbf{F}_{B}$ as marginals can be obtained from Cohen's class TFDs as $(\mathcal{V C U} s)(a, b)$, where $(\mathcal{V C U} s)(x, y)=|\dot{v}(x)|(\mathcal{C U} s)(v(x), y)$. These distributions localize signals along group delays equal to that of $\mathcal{Z}^{-1} \mathbf{e}_{t}^{T}$ and along instantaneous frequencies equal to that of $\mathcal{U}^{-1} \mathbf{e}_{f}^{F}$.

The proof of this result follows by direct substitution in the integrals of $(\mathcal{V C U} s)(a, b)$ with respect to $a$ and $b$. Note that the transformations $\mathcal{U}$ and $\mathcal{Z}$ need not be axis warping operators themselves. Similar results hold for doubly warped affine classes, the primary departure from the above theory being different covariance properties.

Double warping can generate many new joint distribution classes, including all the examples used in $[8,9]$ for illustrating more complicated general techniques. In particular, all distributions with marginals of time and transforms of the form $\left(\mathbf{F}_{K} s\right)(k)=\int s(x) e^{-j 2 \pi k w(x)}|\dot{w}(x)|^{1 / 2} d x$ fall within its grasp. Each different $\mathbf{F}_{K}$ (the Fourier, Mellin, and chirp $[6,7]$ transforms are three examples) matches a distinct class of instantaneous frequency characteristics.

\section{CONCLUSIONS}

Unitary transformations have given us an interesting new "warped perspective" on time-frequency and time-scale analysis. While perhaps surprising, the power of the cascade in Fig. I should not be underestimated, because this simple processing at once obviates piecemeal generalizations of signal analysis tools. In particular, double warping can generate distributions that localize on almost arbitrary group delay and instantaneous frequency characteristics. Since unitary transformations merely change bases, study of $\mathcal{V U}$-Cohen's and $V \mathcal{V}$-affine classes is easy: each property of Cohen's class and the affine class simply transforms in a one-to-one fashion to a corresponding property in the warped class [6, 7].

In this paper we have considered but two methods for coordinating pre- and postprocessing transformations to generate new distributions; other methods should expand the already extensive possibilities even further. So large is the number of new distribution classes attainable through double warping that more general methods may not be required for quite some time. Furthermore, any tools outside the reach of warped Cohen's and affine classes will immediately seed new warped classes of distributions.

\section{REFERENCES}

[1] L. Cohen, "Time-frequency distributions - A review," Proc. $I E E E$, vol. 77, pp. 941-981, July 1989.

[2] O. Rioul and P. Flandrin, "Time-scale energy distributions: A general class extending wavelet transforms," IEEE Trans. Signal Processing, vol. 40, pp. 1746-1757, July 1992.

[3] A. Papandreou, F. Hlawatsch, and G. F. Boudreaux-Bartels, "The hyperbolic class of quadratic time-frequency representations," IEEE Trans. Signal Processing, vol. 41, pp. 34253444, Dec. 1993.

[4] F. Hlawatsch, A. Papandreou, and G. F. Boudreaux-Bartels, "The power classes of quadratic time-frequency representations," in Proc. 27th Asilomar Conf., pp. 1265-1270, 1993.

[5] R. G. Baraniuk and D. L. Jones, "Shear madness: New orthonormal bases and frames using chirp functions," IEEE Trans. Signal Processing, vol. 41, pp. 3543-3548, Dec. 1993.

[6] R. G. Baraniuk and D. L. Jones, "Warped wavelet bases: Unitary equivalence and signal processing," in Proc. ICASSP '93, vol. III, pp. 320-323, 1993.

[7] R. G. Baraniuk and D. L. Jones, "Unitary equivalence: A new twist on signal processing," IEEE Trans. Signal Processing, 1993, Preprint \& Tech. Report 9310, Dept. ECE, Rice Univ.

[8] L. Cohen, "The scale representation," IEEE Trans. Signal Processing, vol. 41, pp. 3275-3292, Dec. 1993.

[9] R. G. Baraniuk, "Beyond time-frequency analysis: Energy densities in one and many dimensions," in Proc. ICASSP' 94 , vol. III, pp. 357-360, 1994.

[10] A. M. Sayeed and D. L. Jones, "Signal transforms covariant and invariant to operators," IEEE Trans. Signal Processing, 1994, Preprint.

[11] R. G. Baraniuk and D. L. Jones, "New dimensions in wavelet analysis," in Proc. ICASSP '92, vol. V, pp. 137-140, 1992.

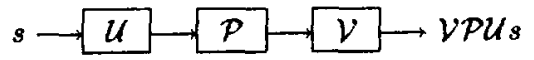

Figure 1: The prototype unitarily equivalent system.

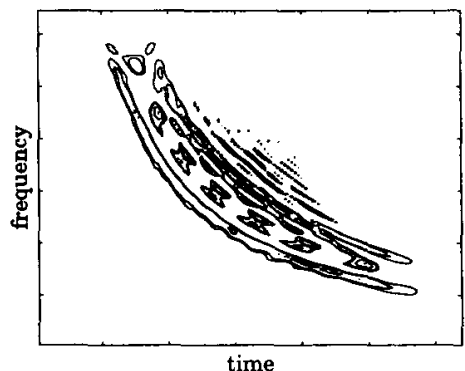

Figure 2: Wigner distribution of the test signal $U_{\log }^{-1} \mathrm{e}_{h}^{T}$ from (3).

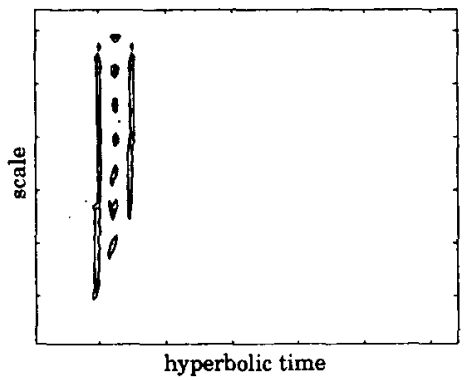

Figure 3: $U_{\log }-W i g n e r(Q)$ distribution of the test signal $\mathcal{U}_{\log }^{-1} \mathbf{e}_{h}^{T}$ from (3).

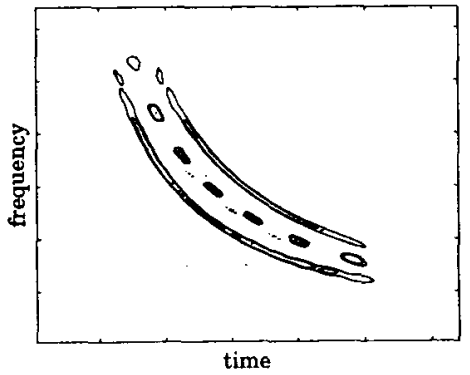

Figure 4: $\mathcal{V}_{\text {log }} \mathcal{U}_{\text {log }}-$ Wigner distribution $(Q$ $T F D$ ) of the test signal $U_{\mathrm{log}}^{-1} \mathbf{e}_{h}^{T}$ from (3). 